\title{
ANN Based 1-bit Full Adder-SubtractorCircuit using Analog
}

\section{Inputs}

\author{
Hassan Mushtaq and Ahsan Rahman
}

Dept. of Computer Science - SZABIST (Dubai Campus) U.A.E., +971-50-4895187, ahsan@szabist.ac.ae

\section{ABSTRACT}

In this paper, we have designed the detailed logical circuits of 1-bit full adder- subtractor working on analog inputs while manipulating it through half-adder, NAND, AND and OR gates integrating with ANN and Machine Learning algorithm where fixed bias is also applied with each of the designed logical gate combinations. Because hardware implementation is far better than the software manipulation so combination of designed circuit can be used to further design ANN based 4-bit or 8-bit full Adder Subtractor. Creating the new hardware device following the same circuits and techniques can reflect the best performance of Adder Subtractor over analog inputs. Resultant waveform of designed ANN based 1-bit full Adder-Subtractor and its subcircuits are completely in favor of its new hardware implementation.

Key words:Half Adder, Full Adder, Adder-Subtractor, Artificial Neural Network (ANN), Machine Learning, Bias, Weights, Hardware, Logic gates, Simulation.

\section{INTRODUCTION}

Artificial Neural Network has been implementing widely almost in all the areas of industries because of its powerful and extensively effective algorithms and techniques to perform much difficult tasks in the same way as human brain do. Software implementation of ANN has been on its peek with the help of Machine learning tools and algorithms. But the hardware implementation of ANN is going towards skies slowly while integrating it with existing technologies to make them intelligent to work according to the situation without human interference at that moment.

ANNs are mainly software-implemented. That is the advantage, in light of the fact that the planner doesn't have to get familiar with the internal operations of components of neural systems, yet can focus on nerve arrange execution. Nevertheless, in real-time applications a drawback has poor performance compared to hardware-based ANNs based software. Hardware-based ANN is introduced in both Digital and Analog circuits [1].

The hardware created was the product of various specifications and has specific uses for this purpose. Information regarding each circuit would be needed to select the equipment for a given task. Various methods may be effective or dependent on the weighing precision, the maximum load, the form of network implemented, the quality of circuit training algorithms 
DOI: https://dx.doi.org/10.26808//rs.ca.i10v4.01

International Journal of Computer Application (2250-1797)

Issue 10 Volume 4,July-August 2020

and other software used. According to the neuron model suggested by McCulloch \& Pitts (1943) seen in the Fig 1 below it is widely used in ANN with numerous modifications.

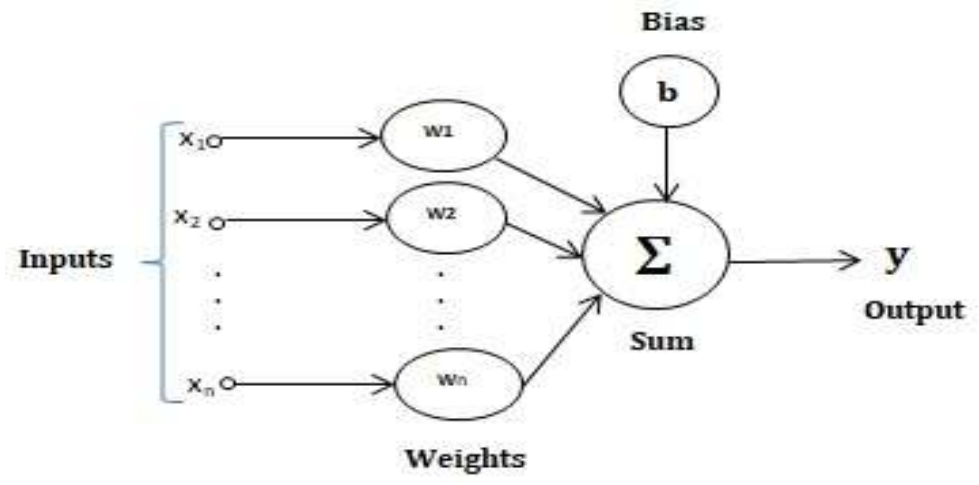

Fig 1: Neuron Model used in ANN

The $\mathrm{N}$ inputs artificial neuron in the Fig 1 appeared as $\mathrm{x} 1, \mathrm{x} 2 \ldots \mathrm{xn}$. Every one of the input is additionally given a weight appeared as $w 1, w 2 \ldots$ wn separately. The bias shown by "b" is likewise adding to summing function. So the actuation work at that point decides if the neuron ought to or ought not to be delivered. The neuron model can be demonstrated in recipe as appeared in Fig 2.

$$
\mathrm{y}=\sum^{n} x i w i+b
$$

Fig 2: Neuron Model's Formula

As the structure of ANN sums multiple inputs along with the weight of each input as well as bias to produce the outputs, in our designed circuit of 1 bit adder-subtractor we also used summing operational amplifier to perform the same methodology of ANN in hardware implementation through logic gates.

The execution of neural systems through hardware has already been examined in a few articles, such as Perez-Unib and Sanchez 1996, Damien et al. 1996, and Molz et al. 2000. According to Moreno Moreno et al. 1999aand Moreno et al. 1999b, a reusable tool was proposed, which can be used to realize most of the arithmetic functions required to implement neural networks in hardware[2].

As is known, two basic hardware models can be considered for hardware implantation projects: analog hardware and digital hardware. Analog hardware can better reproduce nonlinear functions, which are often present in transfer functions of neurons. Digital hardware is not so precise, but provides projects with simplicity and good performance[2].

In general, integrated circuit design is much more complex in case of analog circuits as compared to digital circuits, especially when scaling a large number of gates. It is not different in case of ANN circuit: Analog design can lead to more power and field efficient circuits (Which is not usually the case for traditional ICs where the footprint of the analog part is dominating), their design Cannot be automated to the same extent as digital Circuit. 
DOI: https://dx.doi.org/10.26808//rs.ca.i10v4.01

International Journal of Computer Application (2250-1797)

Issue 10 Volume 4,July-August 2020

Because it is inherently more difficult to scale Analog circuits are more general design circuits Difficult to achieve; however, for the given application, analog Circuits can be much more efficient than their digital Counterparts[3].

In this work, we implemented an ANN model in circuitry implementation of 1-bit adder subtractor through analog hardware while using the logic gates, operational amplifier and their combinations.

\section{LITERATURE REVIEW}

To take advantage of the large scale scalability offered by artificial neural networks, hardware implementations are needed. Most basic neural network models, however, are not quite good for hardware execution, and require optimization. This section offers a summary of the numerous problems that emerged when translating an optimal neural network model to a compact and stable implementation of neural hardware [4].

Logic gates are considered as backbone in designing and building the electronic circuits to make all the electronic equipment operate able and useful. Almost all the circuits are combinational where basic logic gates OR, AND, NOT are combined to design and build complex and advanced logic circuits NOR, NAND, XOR, XNOR, Counters, Buffers, CMOS, Adders, Adder-Subtractor, Multiplexer etc. NOR or NAND that are said to be universal gates that can be used to design and build any multipurpose combinational circuits.

The basic components may be suggested in several forms, including neural-logic AND, OR, and NOT gates. Because various applications have different characteristics, each application may not be handled well by a conventional neural network with a standard structure if certain network connections are redundant and trigger internal disruptions which mean training and network efficiency[5].

Although all the combinational circuits have their own effectiveness, usage and require different ways to design, Full Adder and Subtractor have its own effectives towards achieving useful results in electronics and computer architecture.

A full Adder-subtractor is a type of circuit that can perform addition or subtraction of numbers (especially, binaries). Though is a circuit that adds or subtracts a control signal based on that. A circuit may also be designed that executes both addition and subtraction simultaneously.

4-bit and 8-bit adder-subtractor has already been used in building multiple electronic circuits to get the required outputs. This circuit combines both addition and subtraction operations.

1 bit adder-subtractor is generally utilized in structures, for example, counters, joints, deduction, increment, division, and so on. It is the Arithmetic-Logic-Unit's (ALU) principal part. Also, the innovation and speeding up of FAIS infers the pace of the focal processor-unit (CPU) and the yield of the entire gadget specifically. The FA/S is a focal cell in the CPU and is essential to such an extent that it is difficult to alter. This doesn't, in any case, preclude scientists from endeavoring to expand FAIS pace. A common FAIS depends on three gate types, for example, XOR, AND and OR. FAIS has additionally been structured utilizing CMOS and Mux [6]. 4-bit parallel full adder-subtractor is as appeared in Fig 3. 


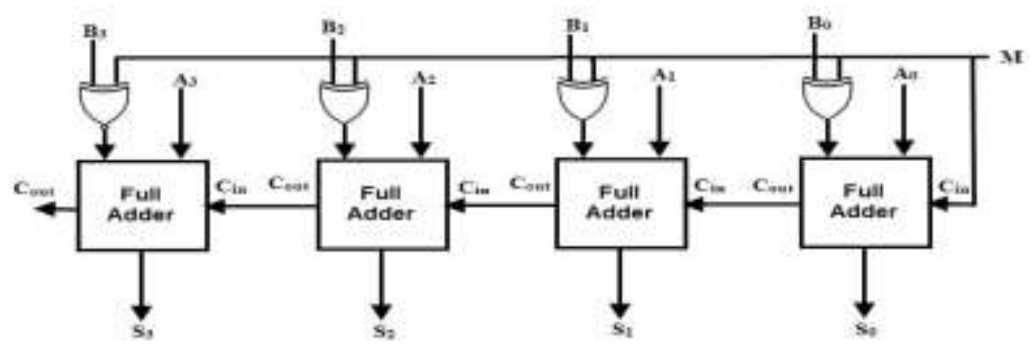

1-bit full Adder-Subtractor has been manipulated in this research work where basic logic gates have been used to design the complete circuits of NAND, AND, OR, Half Adder and full Adder Subtractor to implement it through Artificial Neural Network algorithm.

\section{THE PROPOSED ANN BASED 1-BIT FULL ADDER-SUBTRACTOR CIRCUIT USING ANALOG INPUTS}

Artificial Intelligence has taken the world to new heights of success in term of providing ease to humans in every field of life. AI has been adding intelligence to almost all existing products. AI is not considered as an individual application in most cases, rather it helps to improve the product capabilities like Bixby and Siri features in Samsung and Apple respectively. Both versions employ $\mathrm{AI}$ algorithms and principles to execute various voice commanded tasks. And Robotics, conversational phases, bots and smart robots can also be paired with a lot of knowledge to develop various technologies at home and in the work world, from protection expertise to hypothesis study.

Artificial intelligence achieves mind blowing precision by Artificial Neural Network - which was unimaginable before. Of starters, the links with Alexa, Google Search and Google Photos are built solely on deep learning-so the more we use them, the more slowly they are accurate. Throughout the clinical area, it will now be feasible to use AI techniques through profound understanding, image arrangement, and object identification to discover malignant growth on MRIs with comparable specificity to radiologists who are extraordinarily trained.

The creativity is changing projects from account to assembly of different products, processes and capacities as company leaders and innovators compete to arrive at the promise of manmade consciousness to express an upper hand much as expense and time reserve assets.

Now a day, most of the work is being done in term of Software to create the software side applications using Artificial Neural Network but their hardware implementation is not much.ANN algorithms and techniques are also been implementing in the field of electronics like creating logic gates, half adder, full adder etc.

While using the ANN techniques, we have created the electronic circuit of 1-bit full adder which rectifies that the performance through hardware implementation is much better than software implementation. So we tried to implement ANN techniques using hardware logic gates.

\subsection{CIRCUIT DESIGNING OF NAND GATE}

In logic gates, NAND gate is having special importance for being universal gate. As all the logic gates like OR, AND, NOT, NOR etc. can be made using NAND gate, so its importance to create any logical electronic circuit is more than others. 
DOI: https://dx.doi.org/10.26808//rs.ca.i10v4.01 International Journal of Computer Application (2250-1797)

Issue 10 Volume 4,July-August 2020

The symbol of NAND gate with 2 inputs that is used normally in logic circuit is as Fig 4 is showing.

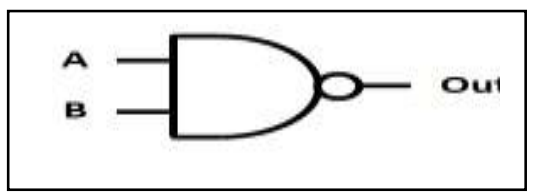

Fig4: NAND gate with 2 inputs

While NAND Gate's truth table is represented in table1:

Table1. NAND gate's truth table

\begin{tabular}{|c|c|c|}
\hline \multicolumn{2}{|c|}{ Inputs } & Output \\
\hline A & B & Out \\
\hline 0 & 0 & 1 \\
\hline 0 & 1 & 1 \\
\hline 1 & 0 & 1 \\
\hline 1 & 1 & 0 \\
\hline
\end{tabular}

As shown, NAND gate gives positive output " 1 " when any of the input is positive "1". And it gives negative output " 0 " when both the inputs are positive.

To improve the performance, NAND gate can also be designed using the combinations of Op-Amp (Operational Amplifier) and set of registers as shown in Fig 5.

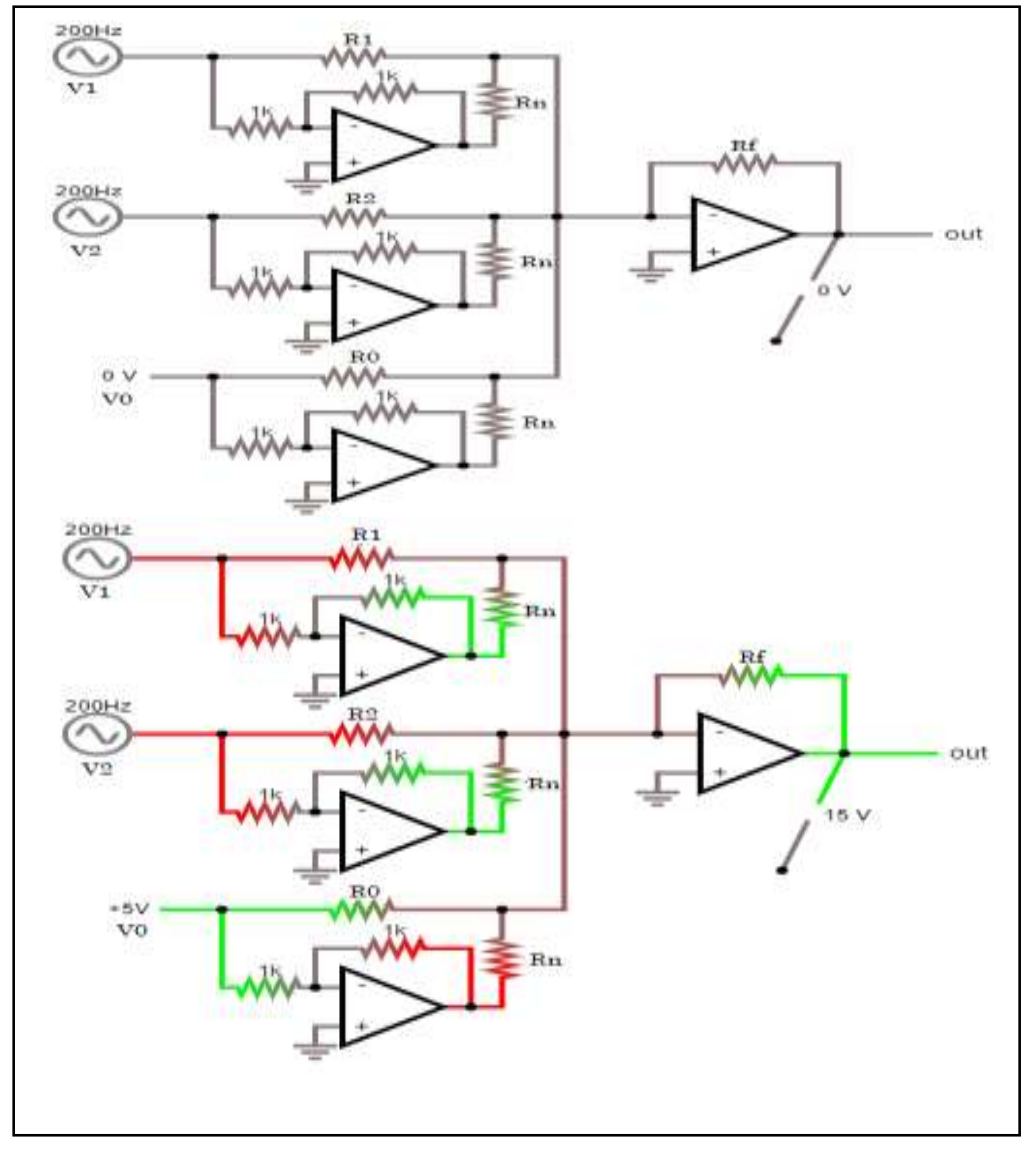

Fig5: NAND gate's circuit using Op-Amp and registers 
DOI: https://dx.doi.org/10.26808//rs.ca.i10v4.01 International Journal of Computer Application (2250-1797) Issue 10 Volume 4,July-August 2020

In this designed circuit V1 and V2 are two inputs and V0 is used as bias input that is kept fixed. The "Rn" that is actually -ve resistor utilized in this designed circuit is determined to be $18.182 \mathrm{~K} \Omega$. What's more, the $\mathrm{Rf}$ is feedback resistor and is kept $222.22 \mathrm{~K} \Omega$ [7].

To implement the functionality of NAND gate, the resistance values of R1, R2 and R0 is taken as $11.713 \mathrm{~K} \Omega, 12.365 \mathrm{~K} \Omega$ and $38.386 \mathrm{~K} \Omega$ respectively[7].

While applying the calculated resistances, the circuitry of NAND gate becomes like as Fig 6 .

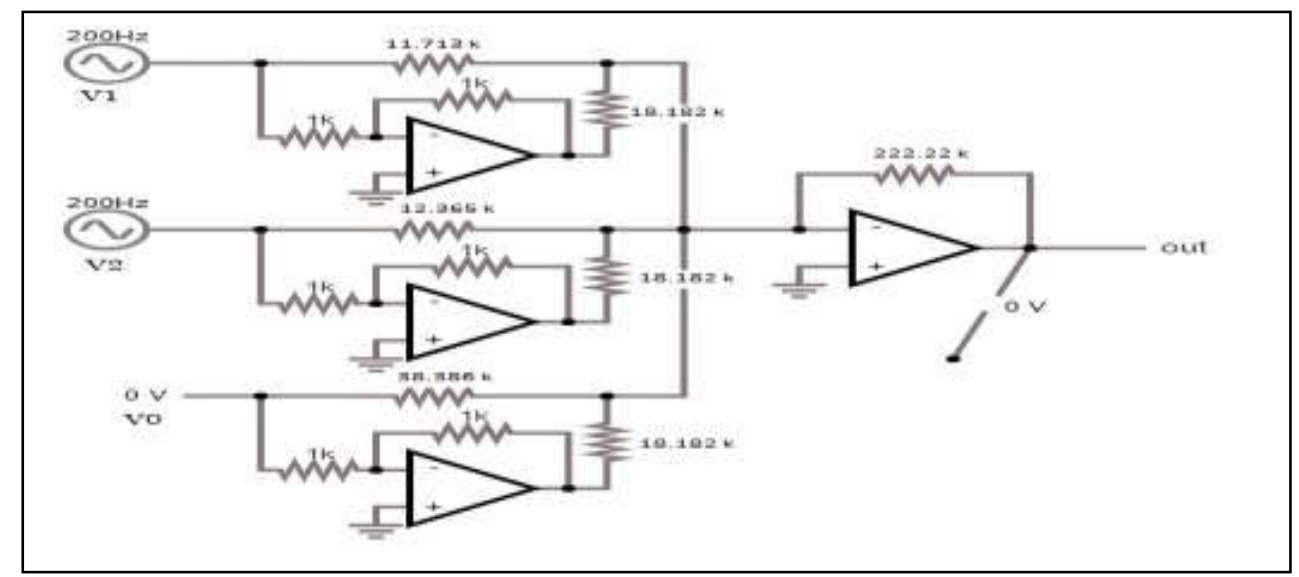

Fig 6: Values assigning to designed NAND gate's circuit

After simulation of the designed NAND gate with $5 \mathrm{~V}$ A/C inputs and fixed bias $+5 \mathrm{~V} \mathrm{D} / \mathrm{C}$; the results are shown in below Fig 7.

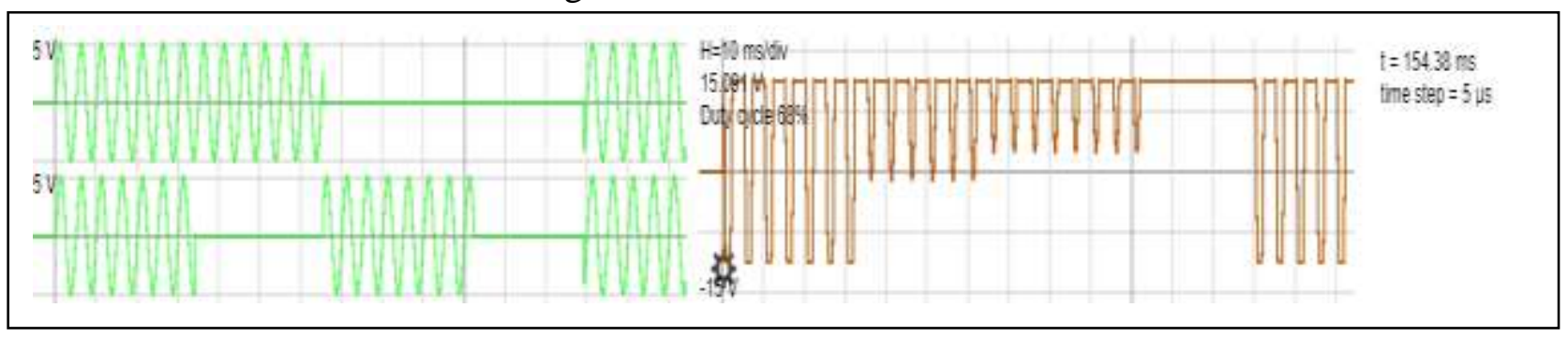

Fig7: Simulation results of designed NAND gate

This circuit attached with some resistance is working correctly for NAND gate where two $5 \mathrm{~V}$ $\mathrm{A} / \mathrm{C}$ inputs and $+5 \mathrm{~V} \mathrm{D} / \mathrm{C}$ bias are generating $15 \mathrm{~V}$ output. Also the role of bias is also shown while both the inputs are zero; bias is the reason for given positive output.

\subsection{CREATING XOR'S CIRCUIT FROM NAND GATE}

As XOR is not from basic gates so it is designed from basic logic gates. So the design of XOR from NAND gate is as shown below in Fig 8:

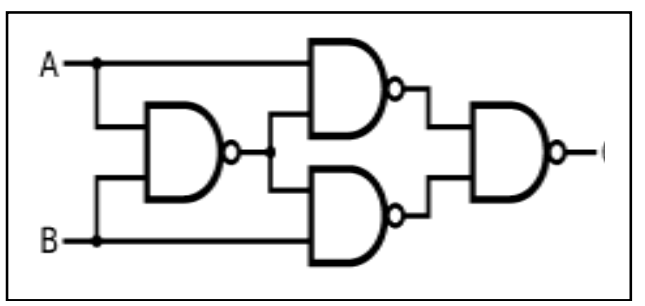

Fig 8: XOR gate designed from NAND gate 
DOI: https://dx.doi.org/10.26808//rs.ca.i10v4.01 International Journal of Computer Application (2250-1797) Issue 10 Volume 4,July-August 2020

Also, the truth table of XOR is as shown by table2.

Table2: XOR's truth table

\begin{tabular}{|c|c|c|}
\hline \multicolumn{2}{|c|}{ Input } & Output \\
\hline A & B & Out \\
\hline 0 & 0 & 0 \\
\hline 0 & 1 & 1 \\
\hline 1 & 0 & 1 \\
\hline 1 & 1 & 0 \\
\hline
\end{tabular}

Following the same model, XOR circuit is designed using the designed NAND gate as shown in Fig 9.

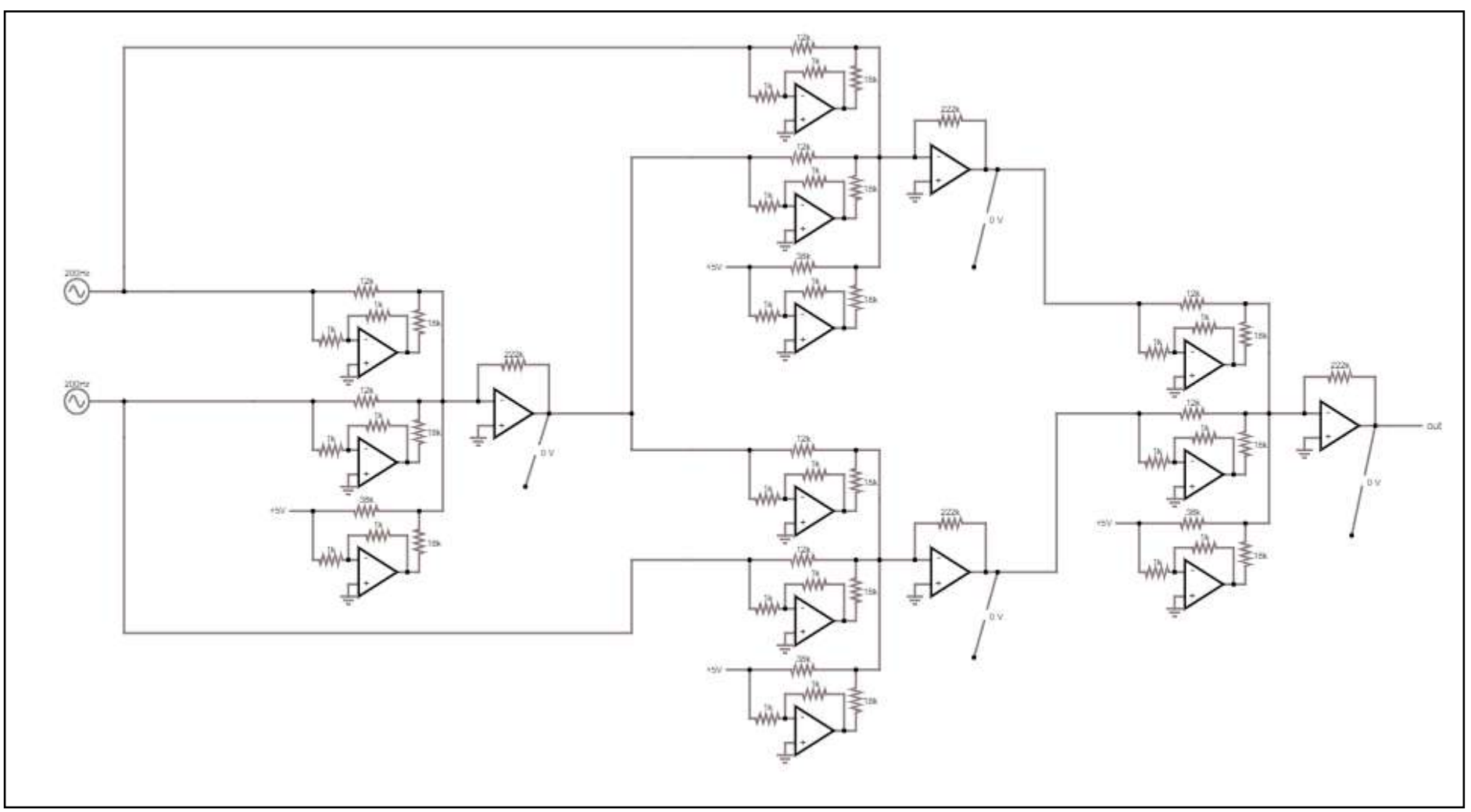

Fig 9: XOR's circuit designed from NAND gate

After simulation, the output waveform is exactly same that is showing its correctness according to the XOR truth table. The role of bias is clear when both the "0" inputs. Fig 10 is showing the output.

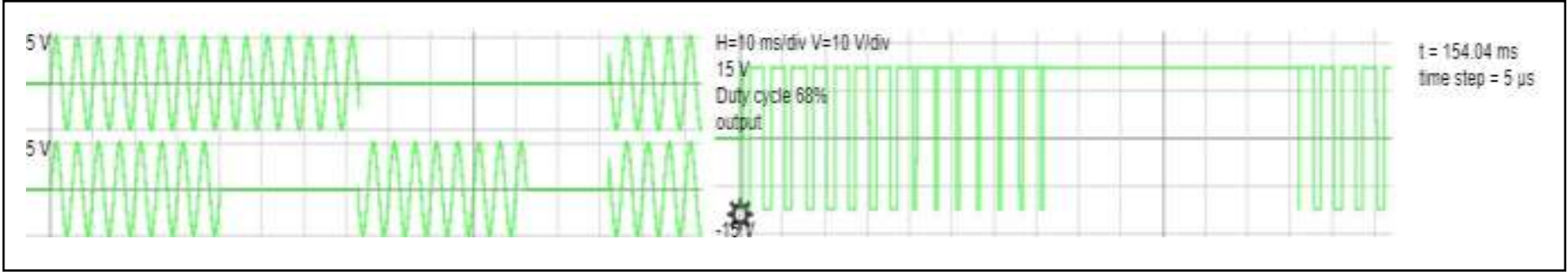

Fig 10: Simulation results of designed XOR gate 
DOI: https://dx.doi.org/10.26808//rs.ca.i10v4.01 International Journal of Computer Application (2250-1797) Issue 10 Volume 4,July-August 2020

\subsection{CREATING 1 BIT HALF ADDER'S CIRCUIT USING XOR AND NAND GATES}

Now to create Half-Adder having the diagram in Fig 11, the combination of XOR and NAND is used as shown in Fig 12.

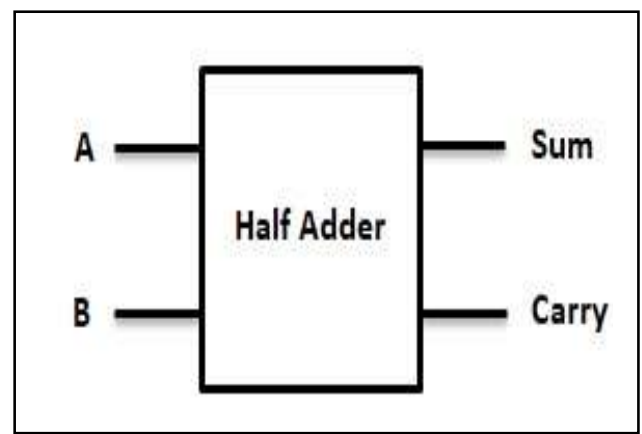

Fig 11: Half Adder diagram

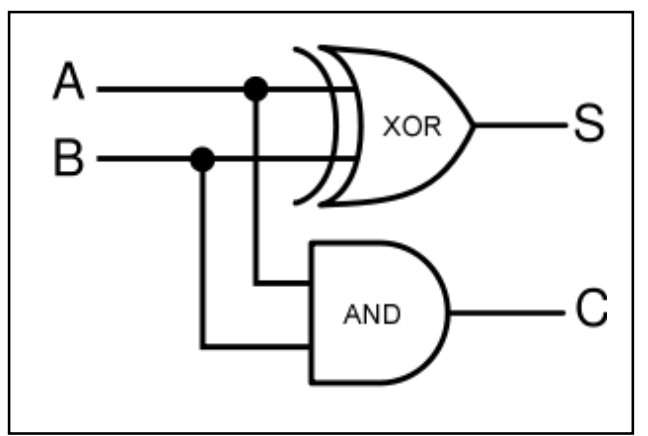

Fig 12: Half Adder designed from NAND gate

The truth table of Half-adder is as presented in table3.

Table3.Truth table of Half-Adder

\begin{tabular}{|c|c|c|c|}
\hline \multicolumn{2}{|c|}{ Inputs } & \multicolumn{2}{c|}{ Output } \\
\hline A & B & Out/Sum & Carry \\
\hline 0 & 0 & 0 & 0 \\
\hline 0 & 1 & 1 & 0 \\
\hline 1 & 0 & 1 & 0 \\
\hline 1 & 1 & 0 & 1 \\
\hline
\end{tabular}

The accompanying half adder circuit Implementation utilizing the XOR and NAND gates shown above is as appeared underneath in Fig13.

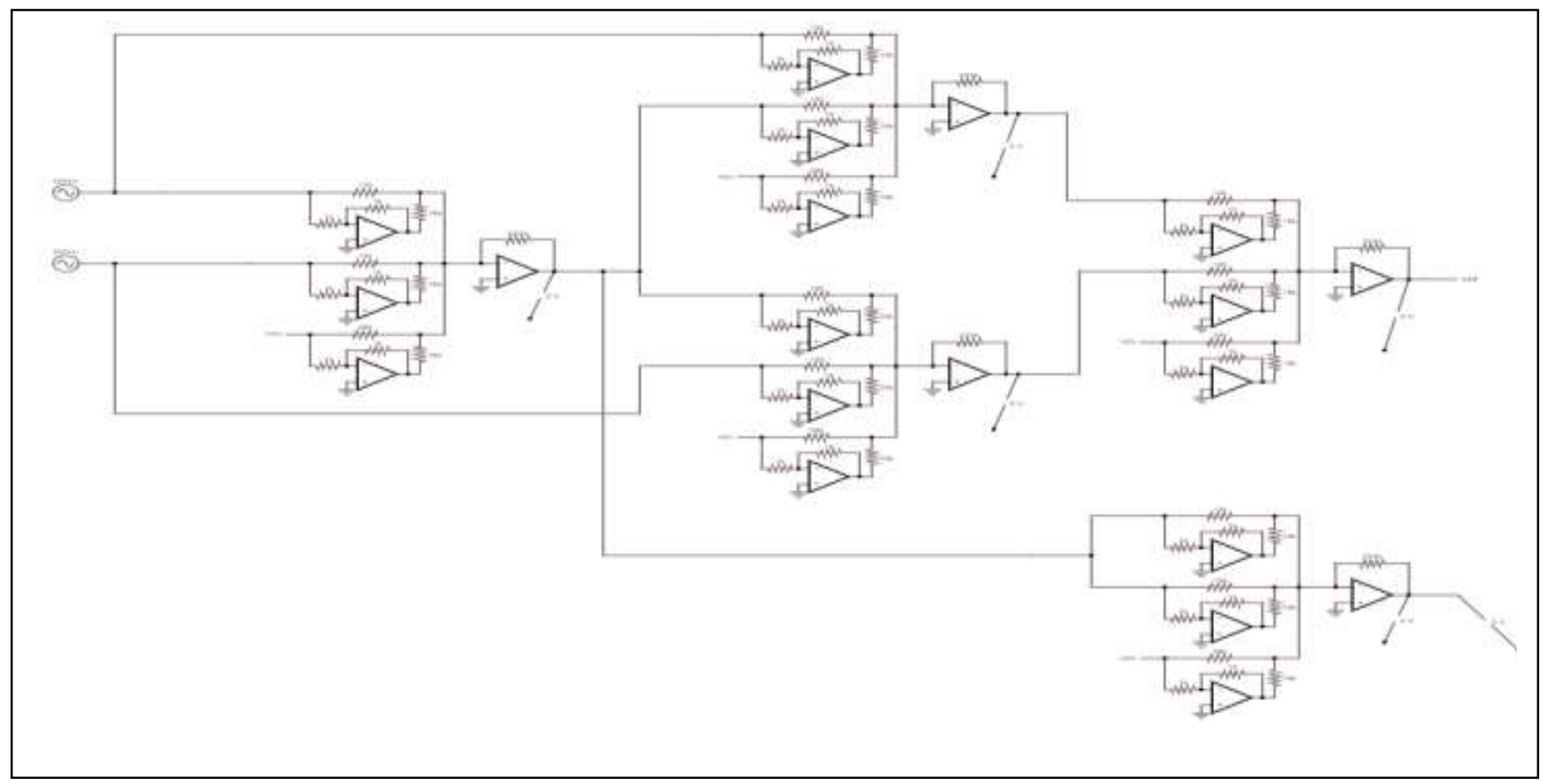

Fig 13: Half-Adder's circuit designed from NAND gate 
DOI: https://dx.doi.org/10.26808//rs.ca.i10v4.01 International Journal of Computer Application (2250-1797) Issue 10 Volume 4,July-August 2020

- The output waveformof "SUM"from the designed half-adder is as shown in Fig 14.

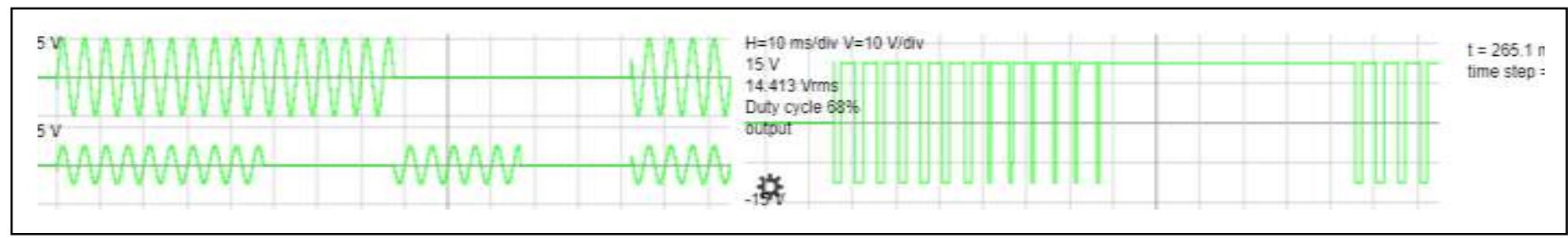

Fig 14: Simulation results of designed Half-Adder from 'SUM'

- The output waveform of "Carry" is shown below in Fig15.

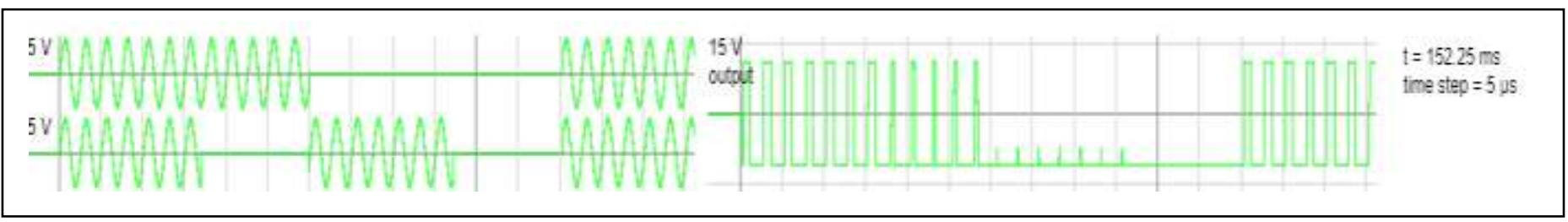

Fig 15: Simulation results of designed Half-Adder from 'Carry'

The output from "OUT" and "CARRY" of designed half-adder circuit is correctly matching with its truth table. Also it is showing the role of bias when both the inputs are " 0 " then only bias is used for results.

\subsection{CREATING 1 BIT FULL ADDER-SUBTRACTOR}

The basic diagram of 1 bit full adder is as shown by Fig 16, while Fig 17 is showing the Full Adder-Subtractor designed from XOR and NAND gates.

$$
\begin{array}{c|c|c|}
\hline \text { B } & \text { Full Adder } & \text { surn } \\
\hline \text { Carry in } &
\end{array}
$$

Fig16: Half Adder-Subtractor diagram

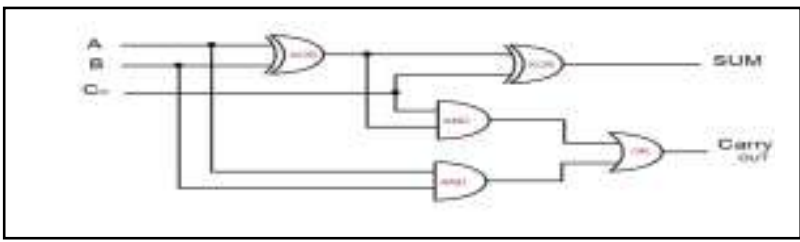

Fig17: Full Adder-Subtractor designed from XOR and NAND gate

The truth table of 1 bit Full Adder-subtractor is shown by table4.

Table4. 1-bit Full Adder-Subtractor's truth table

\begin{tabular}{|c|c|c|c|c|}
\hline \multicolumn{3}{|c|}{ Inputs } & \multicolumn{2}{c|}{ Output } \\
\hline A & B & Cin & Sum & Carry \\
\hline 0 & 0 & 0 & 0 & 0 \\
\hline 0 & 0 & 1 & 1 & 0 \\
\hline 0 & 1 & 0 & 1 & 0 \\
\hline 0 & 1 & 1 & 0 & 1 \\
\hline 1 & 0 & 0 & 1 & 0 \\
\hline 1 & 0 & 1 & 0 & 1 \\
\hline 1 & 1 & 0 & 0 & 1 \\
\hline 1 & 1 & 1 & 1 & 1 \\
\hline
\end{tabular}


DOI: https://dx.doi.org/10.26808//rs.ca.i10v4.01 International Journal of Computer Application (2250-1797)

Issue 10 Volume 4,July-August 2020

Just the circuits of AND and OR gates are needed to complete the circuit of 1-bit full adder. The AND gate, made by changing resistances, R1, R2 and R0 with $34.33 \mathrm{~K} \Omega, 31.873 \mathrm{~K} \Omega$ and $11.561 \mathrm{~K} \Omega$ respectively into our basic designed diagram.

Whereas OR gate is designed by using NAND gates following below Fig 18 combinations.

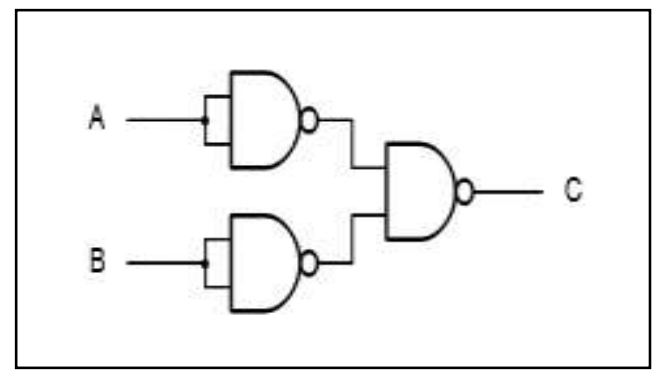

Fig 18: OR gate designed from NAND gates

The final circuit of 1-bit full Adder constructed using the combinations of XOR, NAND and OR gates is as follows in Fig 19.

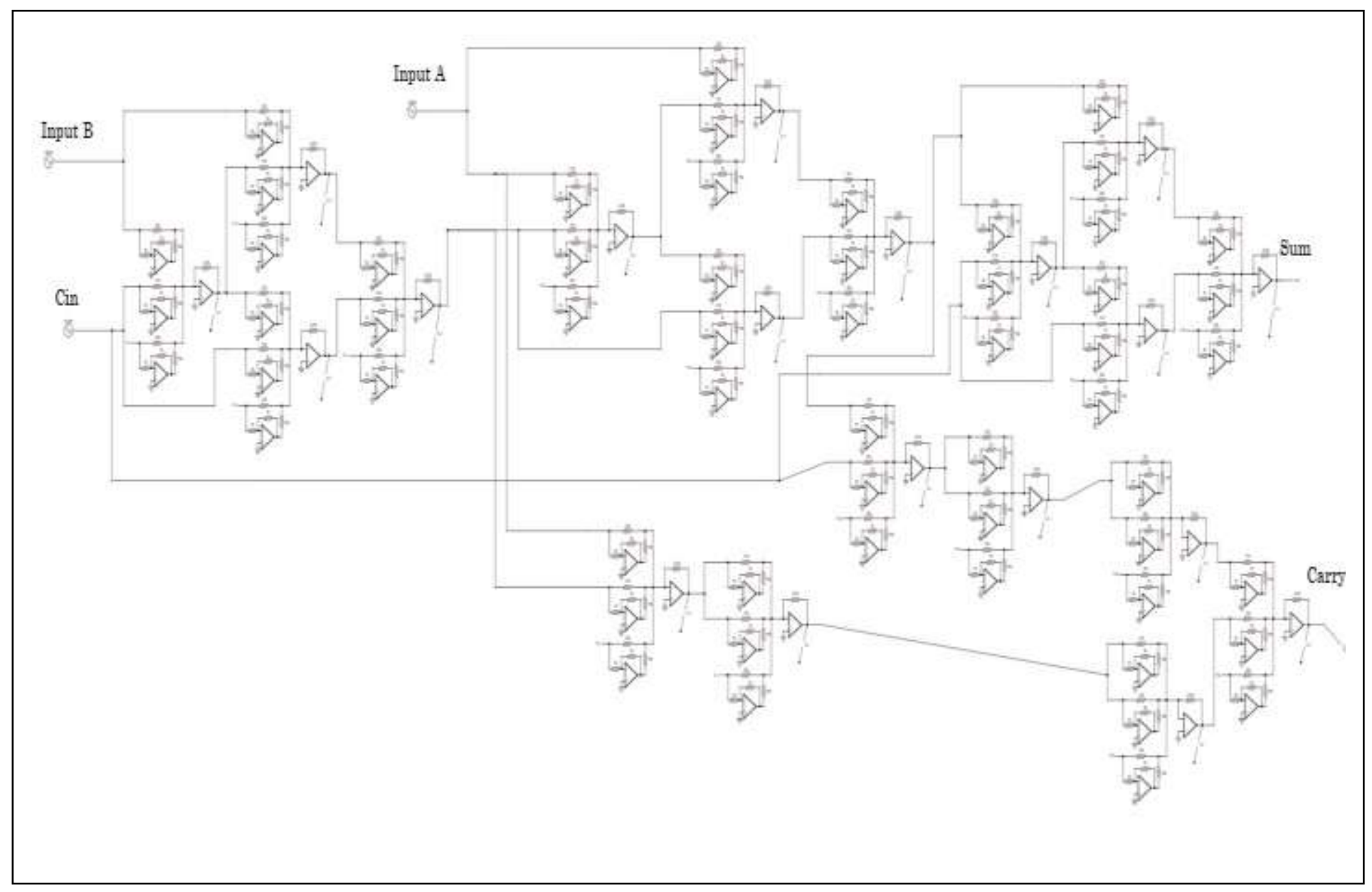

Fig 19: Complete circuit of 1 bit Adder-Subtractor

- The output of "SUM" from above designed 1-bit full adder subtractor is displayed by Fig 20 .

- When "Cin=1(Positive)" 


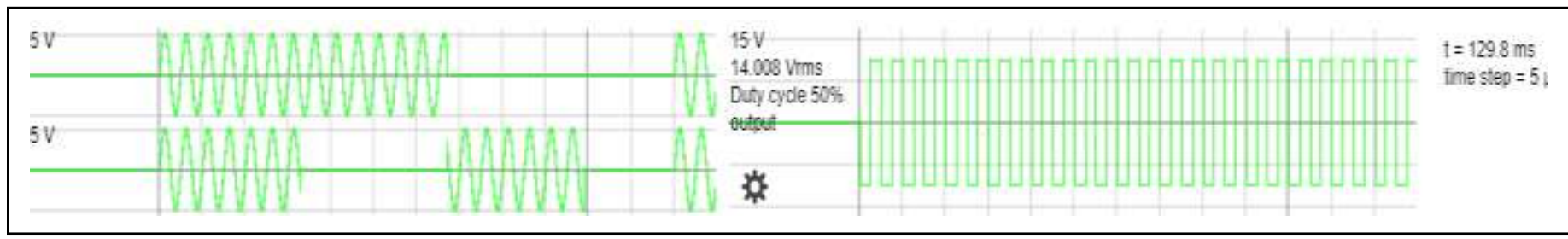

Fig 20: Designed 1 bit Full Adder Subtractor's simulated results from 'SUM' with +ve Cin

- The output of "SUM" from above designed 1-bit full adder subtractor is displayed by Fig 21.

When "Cin=0 (Negative)"

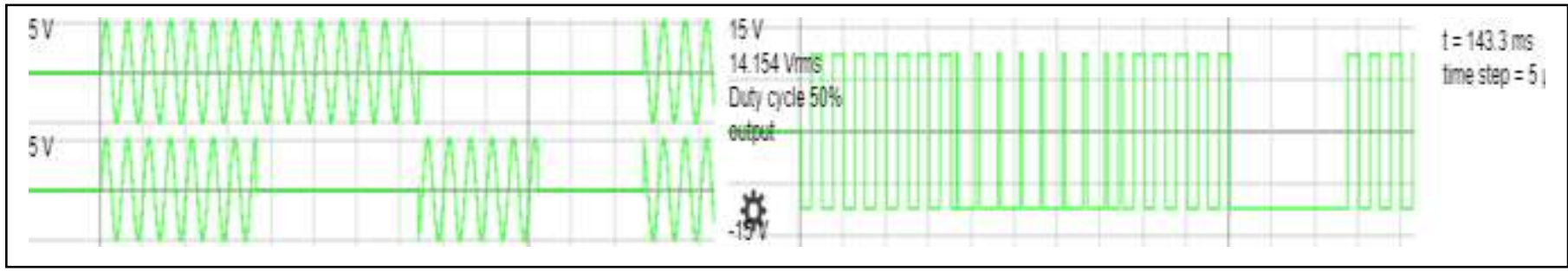

Fig 21: Designed 1 bit Full Adder Subtractor's simulated results from 'SUM' with -ve Cin value

- The output of "Carry/Cout" from above designed 1-bit full adder subtractor is displayed by Fig 22.

When "Cin=1 (Positive)"

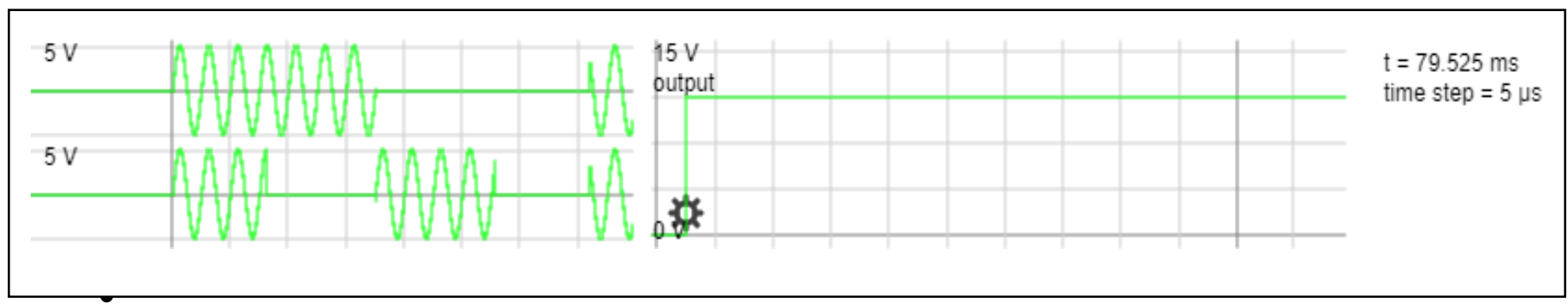

Fig 22: Designed 1 bit Full Adder Subtractor's simulated results from 'Carry’ with +ve Cin value

- The output of "Carry/Cout" from above designed 1-bit full adder subtractor is displayed by Fig 23 .

When "Cin=0 (Negative)"

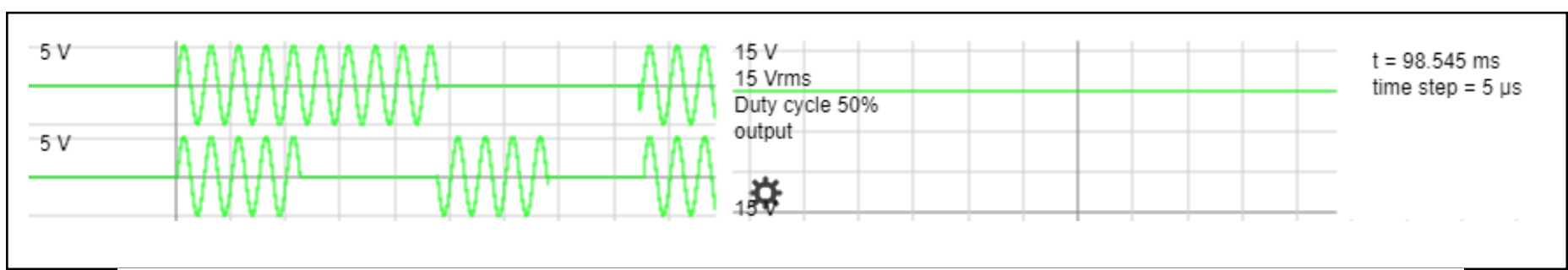

Fig23: Designed 1 bit Full Adder Subtractor's simulated results from 'Carry' with -ve Cin 
DOI: https://dx.doi.org/10.26808//rs.ca.i10v4.01

International Journal of Computer Application (2250-1797) Issue 10 Volume 4,July-August 2020

Subtractor following the ANN algorithm, 4-bit or 8-bit Adder Subtractor detailed circuit can be designedusing the same 1-bit circuit where bias is applied to each logic gate circuit. It can be shaped later in hardware to get the Artificial Neural Network based Adder Subtractor to get the required better results of addition and subtraction simultaneously. Also the activation function can be used to modify the output for specific usage. As analog input $\mathrm{A} / \mathrm{C}$ is giving best performance over digital input $\mathrm{D} / \mathrm{C}$ so maximum output performance can be achieved while created the complete hardware circuit of 4-bit or 8-bit full Adder Subtractor while using the analog input and results can be achieved respectively to make the intelligent electronic equipment.

\section{CONCLUSION}

In this paper, we designed the detailed circuit of 1-bit adder subtractor while following the ANN algorithm and technique where bias is also applied along with the analog inputs of each sub-circuit of 1-bit full adder-subtractor designed in a unique way where maximum output and carry is gained while applying less inputs that can be seen in all the resultant relevant graphs. This can surely help in making the hardware model of Full Adder Subtractor that is having better performance that the software simulation. Multiple combinations of the designed circuit can be used to obtain the detailed circuit of 4-bit or 8-bit ANN model based full Adder-Subtractor that can prove best towards gaining the better equipment intelligence for most effective results.

\section{REFERENCES}

[1] Esraa Z. M. and Haitham K., "Hardware Implementation of Artificial Neural Network", International Journal of Computer Theory and Engineering, vol. 5, no. 5, pp.780-783, 2013.

[2] Denis F. W., Gedson F., Roseli A. F. R., Eduardo M., Marco A. T., Alexandre A. L., Leandro C., Jean M., Rovilson M., "A Pipeline Hardware Implementation for an Artificial Neural Network”, researchgate.net, publication no. 228578786, 2001.

[3] Mats Forssell, "Hardware Implementation of Artificial Neural Networks", Hardware Implementation of Artificial Neural Networks, Ed.859, pp. 1-4,2018.

[4] Perry M. and Emile F., "Neural Network Adaptations to Hardware Implementations", Handbook of Neural Computation, Ed. 1.2, pp. 1-13, 1997.

[5] K.V. Ramanaiah and Siripurapu S., "Hardware Implementation of Artificial Neural Networks", i-manager's Journal on Embedded Systems, vol. 3, no. 4, 2014 - 15.

[6] TranBichThuan P., Yi Wang, Renfa Li, "Designing one-bit Full-Adder/Subtractor based on Multiplexer and LUT's architecture on FPGA", International Journal of Digital Content Technology and its Applications, vol. 7, no. 8, pp. 455-464, 2013.

[7] Aqeeb I. A., Mrinmoy S., Anik C., "INeuromorphic System And Supervised Learning Technique for Visual Pattern Recognition With Memristor Array And CMOS Neuron”, B.S. thesis, Bangladesh University Of Engineering And Technology, 2016. 